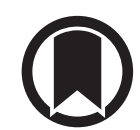

CrossMark

\title{
Training curriculum for European thoracic surgeons: a joint initiative of the European Society of Thoracic Surgeons and the European Respiratory Society
}

\author{
Gilbert Massard ${ }^{1}$, Nathalie Tabin ${ }^{2}$, Lars Konge ${ }^{3}$, Anna Elisabeth Frick ${ }^{4}$, \\ Hasan Batirel ${ }^{5}$, Alessandro Brunelli ${ }^{6}$, Stefano Elia ${ }^{7}$, Martin Huertgen $^{8}$, \\ Laureano Molins (1) $^{9}$, Kostas Papagiannopoulos ${ }^{6}$, Dragan Subotic ${ }^{10}$, \\ Paul van Schil ${ }^{11}$, Gonzalo Varela ${ }^{12}$ and Dirk van Raemdonck ${ }^{13}$
}

Affiliations: ${ }^{1}$ Dept of Medical Education, University of Luxembourg, Luxembourg, Luxembourg. ${ }^{2}$ European Respiratory Society, Lausanne, Switzerland. ${ }^{3}$ Copenhagen Academy for Medical Education and Simulation, Copenhagen, Denmark. ${ }^{4}$ Dept of Thoracic Surgery, Vienna General Hospital, Vienna, Austria. ${ }^{5}$ Dept of Thoracic Surgery, Marmara University Medical School, Istanbul, Turkey. ${ }^{6}$ Dept of Thoracic Surgery, St James's University Hospital, Leeds, UK. ${ }^{7}$ Dept of Thoracic Surgery, University of Rome Tor Vergata, Rome, Italy. ${ }^{8}$ Clinic of Thoracic Surgery, Catholic Clinic Koblenz-Montabaur, Koblenz, Germany. ${ }^{9}$ Respiratory Institute, Barcelona, Spain. ${ }^{10}$ University Hospital Basel, Basel, Switzerland. ${ }^{11}$ Dept of Thoracic and Vascular Surgery, Antwerp University Hospital and Antwerp University, Antwerp, Belgium. ${ }^{12}$ Salamanca Institute of Biomedical Research, Salamanca, Spain. ${ }^{13}$ Dept of Thoracic Surgery, University Hospitals Leuven, Leuven, Belgium.

Correspondence: Gilbert Massard, Dept of Medical Education, University of Luxembourg, 2, avenue de l'Université, L-4365 Esch-sur-Alzette, Grand-Duchy of Luxembourg. E-mail: gilbert.massardauni.lu

@ERSpublications

This consensual curriculum for thoracic surgeons intends to promote homogeneity of training and and its outcome in terms of competence throughout Europe http://bit.ly/3a2tTZI

Cite this article as: Massard G, Tabin N, Konge L, et al. Training curriculum for European thoracic surgeons: a joint initiative of the European Society of Thoracic Surgeons and the European Respiratory Society. Eur Respir J 2020; 55: 1902012 [https://doi.org/10.1183/13993003.02012-2019].

In the most recent issue of the European Journal of Cardio-Thoracic Surgery, the joint task force group on training in thoracic surgery, set up by both the European Society of Thoracic Surgeons and the European Respiratory Society (ERS), has published the core document describing the recommended training curriculum [1]. This document takes its roots from the European syllabus published in 2018 [2, 3]. According to a methodology validated by education scientists, the working group has described, for each item, the expected level of competence in terms of knowledge, skills and attitudes, listed possible learning resources, and recommended appropriate tools for assessment of learning outcomes [4]. The task force group utilised Bloom's taxonomy to characterise learning domains and Miller's model of competence for clinical skills $[5,6]$. Attitudes and non-technical skills were described according to the CanMEDS physician competency framework edited by the Royal College of Physicians and Surgeons of Canada $[7,8]$.

The document has been validated after an internal and external reviewing process.

This curriculum is mainly intended to serve as a guideline to training programmes; programme directors may accordingly organise rotations in more specialised departments for issues such as tracheal surgery, oesophageal procedures, lung transplantation, paediatric thoracic surgery and other rare procedures. 
The optional modules reflect most of these less frequent procedures, whereas more routine thoracic surgery is described in the mandatory modules. Thoracic units have been classified into standard units and highly specialised units [9].

The other targeted readership of this curriculum is composed of thoracic surgery trainees. This detailed document represents a roadmap supporting the trainee through the essentials of thoracic surgery training; such a tool may improve the learning process of thoracic surgical residents [10]. Skills modules and items may be especially utilised to construct an appropriate surgical logbook. The curriculum document stands for thoracic surgery alone, knowing, however, that in many countries, other aspects of surgery are included in the training requirements. In at least seven European countries, the specialty certificate is entitled "thoracic and cardiovascular surgery", implicating that part of the training requires rotations through cardiovascular surgical units [2]. Many countries mandate exposure to general surgery, favouring some surgical "general culture" against early over-specialisation [2]. Last but not least, isolated modules, or groups of items may be utilised for continuing professional development by certified thoracic surgeons.

The main motivation leading to the creation of this task force was related to the heterogeneity of thoracic surgical training in Europe. As opposed to North America, each European state applies its own laws and regulations. Accordingly, the name of the specialty, the certifying body, the length and content of training differ from one country to another [2]. In the context of mutual recognition of professional qualifications between European Union member states, which favours European mobility of surgeons, harmonisation of training programmes and training outcomes is desirable to guarantee equal quality of care to all patients [11]. With the same intent of harmonisation, the UEMS (European Union of Medical Specialists) section for thoracic surgery has created a European Board of Thoracic Surgery, which organises a yearly examination for recently graduated specialists. Becoming a fellow of the board signifies that the applicant has shown the competence level expected from a European thoracic surgeon. The curriculum document provides a reference for the expected competence level in each module and item of the syllabus.

The thoracic surgery community took advantage of the vast experience that ERS has accrued over years of curriculum development. The former HERMES initiative (Harmonised Education in Respiratory Medicine for European Specialists), was launched 15 years ago, with a first task force devoted to adult respiratory medicine [12]. Subsequently, ERS developed several other projects, such as paediatric respiratory medicine, respiratory intensive care, sleep medicine, physiotherapy and thoracic oncology, among others. For each task force, education scientists who followed the group up to completion initially trained the members of the working group in the curriculum design methodology.

What are the challenges for the future? Obviously, knowledge requirements are expanding in many fields. The thoracic surgeon cannot ignore, for instance, new medications and treatment strategies in oncology; this does not exclusively concern lung cancer and mediastinal tumours, because thoracic surgeons are regularly involved with metastases originating from extrathoracic malignancies and frequently attend tumour boards. Diseases and disorders cared for by thoracic surgeons cover many nosological fields and anatomic areas. Further, with ageing populations, we are facing an increased amount and variety of comorbidities, which need to be taken into account for accurate perioperative care. Technology has sped up its development, and many new tools, such as robot assistance, extracorporeal lung support, intrapleural chemotherapy, electromagnetic broncho-navigation, three-dimensional modelling of segmental anatomy, and others, are appearing in the surgeons' environment. Accordingly, this curriculum appears as a contemporary snapshot, but will clearly require revisions and updates in the future.

Another challenge is to put in place mechanisms and processes aimed at continuously monitoring the level of knowledge and skills. While it appears obvious that the acquisition of knowledge and skills should be regularly audited in trainees, it is no less important to test certified consultants as well. Senior surgeons are exposed to fading of expertise and need to acquire new knowledge and skills dictated by scientific and technological developments. It is the duty of our scientific societies to develop appropriate tools of assessment for this purpose.

We intentionally omitted to discuss two specific points. Our curriculum is limited to competence in general thoracic surgery, regardless of whether the individual is trained as a monospecialist in thoracic surgery or as a cardiothoracic surgeon. While we abstained from debating the relative merit of including a cardiac surgical training, the authors agree that some exposure to extracorporeal lung support should be offered to all trainees. The second point is to what extent a thoracic surgeon should be exposed to other surgical specialties, pulmonary medicine, intensive care, etc., reinforcing some "medical and surgical general culture". In the current context, we need to comply with national regulations for both points, as well as for the duration of the training, currently varying from 5-10 years [2]. Finally, learning outcomes are also modulated by the interaction between the individual learning abilities and the opportunities provided by the training programme. 
The authors hope that the present curriculum will be disseminated through national professional organisations and widely utilised by both training programmes and thoracic surgical trainees.

Conflict of interest: G. Massard has nothing to disclose. N. Tabin is an ERS employee. L. Konge has nothing to disclose. A.E. Frick has nothing to disclose. H. Batirel reports personal fees from Johnson and Johnson, outside the submitted work. A. Brunelli has nothing to disclose. S. Elia has nothing to disclose. M. Huertgen has nothing to disclose. L. Molins has nothing to disclose. K. Papagiannopoulos has nothing to disclose. D. Subotic has nothing to disclose.

P. van Schil has nothing to disclose. G. Varela has nothing to disclose. D. van Raemdonck has nothing to disclose.

\section{References}

1 Massard G, Tabin N, Konge L, et al. Training curriculum for European thoracic surgeons: a joint initiative of the European Society of Thoracic Surgeons and the European Respiratory Society. Eur J Cardiothorac Surg 2020; 57: 418-421.

2 Massard G, Tabin N, Mitchell S, et al. A harmonized European training syllabus for thoracic surgery: report from the ESTS-ERS task force. Eur J Cardiothorac Surg 2018; 54: 214-220.

3 Massard G, Tabin N, Mitchell S, et al. A harmonised European training syllabus for thoracic surgery: report from the ESTS-ERS task force. Eur Respir J 2018; 51: 1800370.

4 Harden RM. AMEE Guide $\mathrm{N}^{\circ}$ 21: Curriculum mapping: a tool for transparent and authentic teaching and learning. Med Teach 2001; 23: 123-137.

5 Bloom BS, Engelhart MD, Furst EJ, et al. Taxonomy of educational objectives: The classification of educational goals. In: Handbook I: Cognitive Domain. New York, David McKay Company, 1956.

6 Miller GE. The asessment of clminical skills/competence/performance. Acad Med 1990; 65: 63-67.

7 Frank JR, Snell L, Sherbino J, editors. CanMEDS 2015 Physician Competency Framework. Ottawa, Royal College of Physicians and Surgeons of Canada, 2015.

8 Royal College of Physicians and Surgeons of Canada. CanMEDS: Better standards, better physicians, better care. www.royalcollege.ca/rcsite/canmeds/canmeds-framework-e. Date last accessed: 20 July, 2019.

9 Brunelli A, Falcoz PE, D'Amico T, et al. European guidelines on structure and qualification of general thoracic surgery. Eur J Cardiothorac Surg 2014; 45: 779-786.

10 Antonoff MB, Verrier ED, Allen MS, et al. Impact of moodle-based online curriculum on thoracic surgery in-training examination scores. Ann Thorac Surg 2016; 102: 1381-1386.

11 European Union. Directive 2005/36/EC of the European Parliament and of the Council of 7 September 2005 on the Recognition of Professional Qualifications. Document 32005L0036. http://eur-lex.europa.eu/legal-content/EN/ TXT/?qid=1401715075231\&uri=CELEX\%3A32005L0036

12 Loddenkemper R, Séverin T, Haslam P. European curriculum recommendations for training in adult respiratopry medicine:crossing boundaries with HERMES. Eur Respir J 2008; 32: 538-540. 